\title{
49. Jahrestagung der DGKN
}

Vom 15. bis 19. September findet zum 49. Mal die Jahrestagung der Deutschen Gesellschaft für Klinische Neurophysiologie und Funktionelle Bildgebung (DGKN), diesmal im thüringischen Jena, statt. Der Präsident der 49. Jahrestagung ist der Direktor der Klinik für Neurologie am Universitätsklinikum Jena, Prof. Dr. Otto W. Witte.

Schwerpunkte der Tagung sind das alternde Nervensystem, Epilepsie und zerebrale Plastizität, funktionelle Bildgebung und der Schlaganfall. $\mathrm{Zu}$ diesen Themen gibt es an den fünf Tagen eine Reihe hochkarätiger Symposien und Sitzungen.

Das wissenschaftliche Programm umfasst 34 thematische Sitzungen mit Einzelvorträgen, des Weiteren Hauptvorträge, Mitgliederversammlungen und Seminare. Viele Sitzungen werden durch freie Vorträge ergänzt und durch assoziierte Postersitzungen abgerundet. Acht wissenschaftliche Symposien, u.a. das Symposium "Spastische Spinalparalyse" der Tom Wahlig Stiftung, und neun Satelliten-Symposien renommierter Unternehmen bietet die Tagung den Teilnehmern. Neben dem wissenschaftlichen Programm findet am 18. und 19. September ein umfangreiches Fortbildungscurriculum mit 24 Kursen statt, erstmals ergänzt um Module in dem neuen Curriculum „Funktionelle Bildgebung“. Mit der Verleihung des Fortbildungspreises würdigt die DGKN langjährige
Verdienste um die Fortbildung von Ärztinnen und Ärzten im Bereich der Klinischen Neurophysiologie. Überdies beinhaltet die Jahrestagung auch eigenständige Fortbildungen des Fachverbandes Neurophysiologisch Technischer Assistenten (FNTA) und des Vereins Medizinisch-Technischer Assistenten (MTA). Begleitend bietet die Tagung eine äußerst informative Industrieausstellung mit 60 Partnern an, die ihre Unternehmen und Forschungsergebnisse den Tagungsgästen präsentieren werden.

Besonderer Bestandteil der Jahrestagung ist am 18. September ein Symposium anlässlich des 200-jährigen Bestehens der Jenaer Nervenklinik, die eng mit klangvollen Namen der Neurowissenschaften wie Otto Binswanger und Hans Berger verbunden ist.

Neben den modernen und großzügigen Räumlichkeiten auf dem Campus der Friedrich-Schiller-Universität findet die Tagung auch in der Klinik für Neurologie statt. Diese zog erst Anfang diesen Jahres als erste Klinik in den neugebauten Komplex des Universitätsklinikums. Mit dem Umzug aus dem historischen Gebäude im Stadtzentrum in die modernen Klinikräume verbesserten sich die Bedingungen für Patienten und Mediziner spürbar. „Auch eine Erweiterung des medizinischen Spektrums ist dank der verbesserten Rahmenbedingungen möglich. Vor allem akute Schlaganfälle können noch schneller und effi- zienter behandelt werden", betont Prof. Witte. Eine weitere deutliche Verbesserung stellen die kurzen Wege und die Nähe $\mathrm{zu}$ anderen Fachbereichen, wie dem Nothilfezentrum und der Radiologie, dar - schließlich zählt jede Sekunde bei der Erhaltung wichtiger Gehirnfunktionen. Interessierten Tagungsteilnehmern wird sich sicherlich die Gelegenheit bieten, sich die neu ausgestattete neurologische Intensivstation, die drei neurologischen Stationen und die zusätzliche Tagesklinik anzusehen.

Das Rahmenprogramm der DGKN-Tagung verpasst es nicht den Gästen am Rande der wissenschaftlichen Veranstaltungen einige Aspekte des Thüringer Lebens und Sehenswürdigkeiten von Jena und Umgebung anzubieten. So sind ein Besuch des bekannten Jenaer Glasmuseums, der Schott Villa, des weltberühmten Planetariums und der wunderschönen Dornburger Schlösser ganz in der Nähe von Jena vorgesehen. Ein historischer Stadtrundgang durch Jena, ein Modetag mit Altstadtspaziergang in Thüringens Landeshauptstadt Erfurt und eine Besichtigung der Mahn- und Gedenkstätte Buchenwald runden das Angebot ab. Neue Gesprächspartner und alte Freunde trifft man am Donnerstag zum Thüringer Schwarzbierabend und am Freitag beim Gesellschaftsabend in den 130 Meter über der Stadt gelegenen Räumen des IntershopTowers. 